\title{
Hydroelastic optimization of a keel fin of a sailing boat: a multidisciplinary robust formulation for ship design
}

\section{Matteo Diez, Daniele Peri, Giovanni Fasano \& Emilio F. Campana}

Structural and Multidisciplinary Optimization

ISSN 1615-147X

Volume 46

Number 4

Struct Multidisc Optim (2012)

46:613-625

DOI 10.1007/s00158-012-0783-7

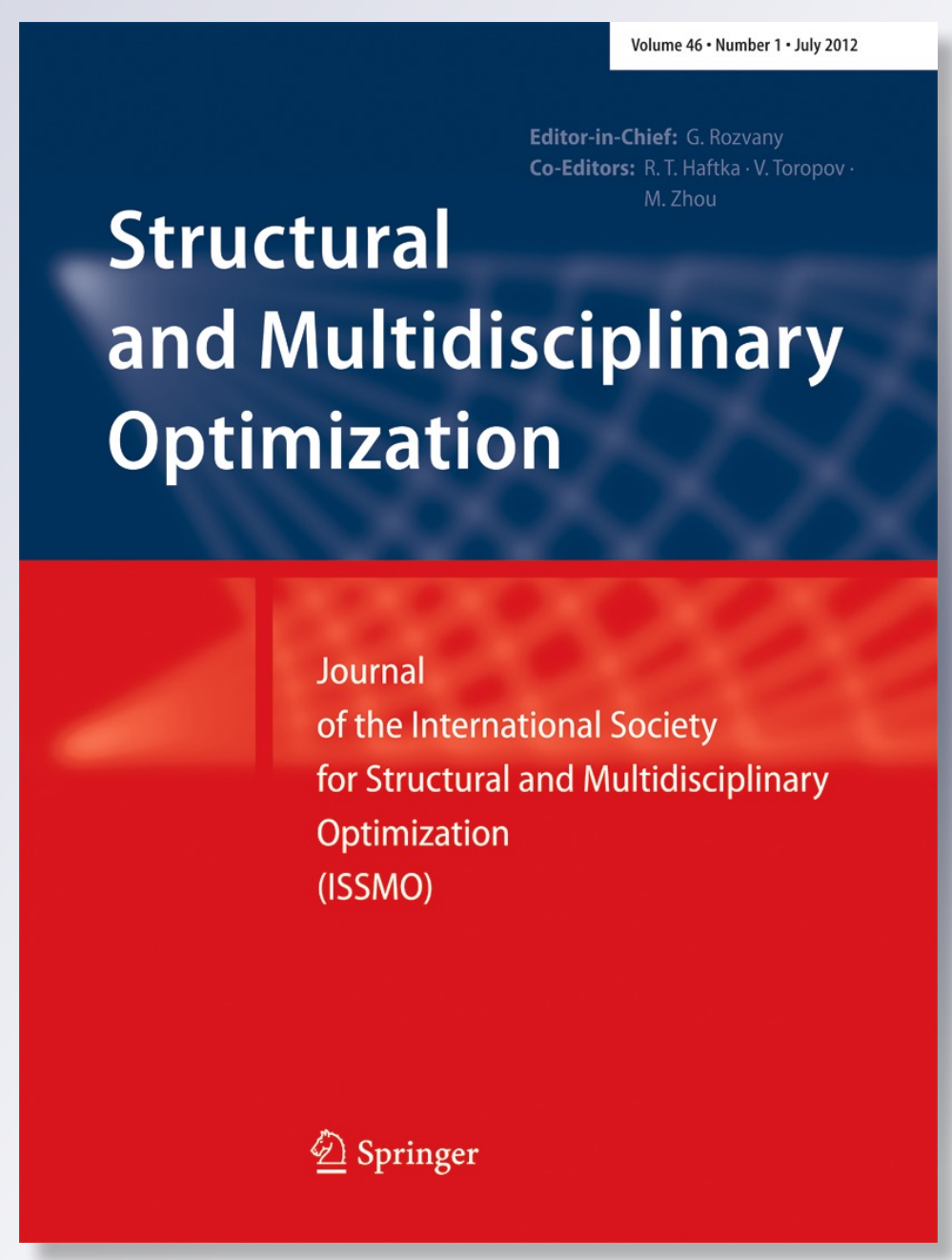

勿 Springer 
Your article is protected by copyright and all rights are held exclusively by SpringerVerlag. This e-offprint is for personal use only and shall not be self-archived in electronic repositories. If you wish to self-archive your work, please use the accepted author's version for posting to your own website or your institution's repository. You may further deposit the accepted author's version on a funder's repository at a funder's request, provided it is not made publicly available until 12 months after publication. 


\title{
Hydroelastic optimization of a keel fin of a sailing boat: a multidisciplinary robust formulation for ship design
}

\author{
Matteo Diez • Daniele Peri · Giovanni Fasano • \\ Emilio F. Campana
}

Received: 15 July 2011 / Revised: 17 December 2011 / Accepted: 15 February 2012 / Published online: 29 March 2012

(C) Springer-Verlag 2012

\begin{abstract}
The paper presents a formulation for multidisciplinary design optimization of vessels, subject to uncertain operating conditions. The formulation couples the multidisciplinary design analysis with the Bayesian approach to decision problems affected by uncertainty. In the present context, the design specifications are no longer given in terms of a single operating design point, but in terms of probability density function of the operating scenario. The optimal configuration is that which maximizes the performance expectation over the uncertain parameters variation. In this sense, the optimal solution is "robust" within the stochastic scenario assumed. Theoretical and numerical issues are addressed and numerical results in the hydroelastic optimization of a keel fin of a sailing yacht are presented.
\end{abstract}

Keywords Multidisciplinary Design Optimization (MDO) · Robust Design Optimization (RDO) .

Hydroelastic optimization · Ship design

This work has been supported by the US Office of Naval Research, NICOP grant no. N00014-08-1-0957, through Dr. Ki-Han Kim. A preliminary version of this manuscript was presented at the 28th Symposium on Naval Hydrodynamics, Pasadena, CA, USA, 2010.

M. Diez $(\varangle)$ · D. Peri · E. F. Campana

CNR-INSEAN, National Research Council-Maritime Research

Centre, Via di Vallerano, 139, 00128 Rome, Italy

e-mail:m.diez@insean.it

G. Fasano

Dip. di Matematica Applicata, Università Ca'Foscari, Ca'Dolfin, Dorsoduro 3825/E, 30123 Venice, Italy

\section{Introduction}

Design optimization formulations and techniques are intended for supporting the designer in the decision making process, relying on a rigorous mathematical framework, able to give the "best" solution to the design problem at hand. Over the years, optimization has been playing an increasingly important role in engineering. Advanced modeling and algorithms in optimization constitute now an essential part in the design of complex aerospace (Hicks and Henne 1978; Sobieszczanski-Sobieski and Haftka 1997; Mohammadi et al. 2001; Alexandrov and Lewis 2002; Willcox and Wakayama 2003; Padula et al. 2006; Morino et al. 2006; Iemma and Diez 2006) and automotive (Baumal et al. 1998; Kodiyalam and SobieszczanskiSobieski 2001) applications. The strong impact of design optimization has attracted the naval community, so that the recent years have seen progress in optimization for ships too (Ray et al. 1995; Peri and Campana 2003, 2005; Parsons and Scott 2004; Pinto et al. 2004; Campana et al. 2007a, b, 2009; Papanikolaou 2009; Diez and Peri 2009, 2010a, b).

Generally, the task of designing a ship (as well as an aerial or ground vehicle) demands that the engineering team considers a host of multidisciplinary design goals and requirements. Multidisciplinary Design Optimization (MDO) classically refers to the quest for the best solution with respect to optimality criteria, whose definition involves a number of disciplines mutually coupled. Therefore, MDO encompasses the interaction of different discipline-systems, formally joined together and inter-connected in a common framework, which leads to a multidisciplinary equilibrium.

In this context, design engineers increasingly rely on computer simulations to develop new designs and to assess their models. However, even if most simulation codes are deterministic, in practice systems' design should be often 
permeated with uncertainty. On this guideline, the most straightforward example in the naval hydrodynamics context is offered by any existing ship, that must perform under a variety of operating conditions (e.g. different, stochastic environmental conditions). Some question arises: how can the results of computer simulations be properly exploited in the framework of design optimization, when the overall context is affected by uncertainty? How can deterministic analysis be integrated in an ad hoc formulation that includes uncertainty? The latter questions stress one of the major issues arising in the optimization of a (ship) design: the perspective from which the optimization problem has to be formulated and performed. Indeed, a "tight" deterministic optimization often leads to specialized solutions that are inadequate to face the "real-life" world, which is instead characterized by a high level of uncertainty. In this respect Marczyk (2000) states that, in a deterministic engineering context, optimization is the synonymous of specialization and, consequently, the opposite of robustness. The perspective we try to give in the present work has the aim of broadening the standard-optimization-problem framing, leading to a formulation in which optimality is recast in terms of robustness, rather than specialization. To the aim of clarifying the latter perspective, it may be useful to summarize the following statements:

- Design optimization is always about answering a question, i.e. assisting the designer in the decision making process.

- Before going through the optimization procedure, special attention has to be paid to the formulation of the problem. In the context of design optimization, inadequate answers often stem from hill-posed questions.

- In this work we try to re-formulate the optimization perspective by looking at the design problem from a broader standpoint. We bring the uncertainty related to ship design, manufacturing and operations, into the decision problem.

- The formulation of the question (we try to answer to, using optimization) relies on optimal statistical decision theory and, specifically, on Bayes principle, defining a rigorous mathematical framework in which the "robust" decision making process is embedded.

In general, in any engineering system the uncertainty is due to variations of design parameters, along with operating or environmental conditions. The uncertainty is also related to the evaluation of the relevant functions, due to inaccuracy in modeling or computing. Using ideas from statistical decision theory, and specifically Bayes principle (De Groot 1970; Trosset et al. 2003; Kugele et al. 2008), the problem of robust decision making in design can be formulated as an optimization problem (Robust Design Optimization, RDO).
In the framework of Bayes theory, we assume that the original "deterministic" design goal is the minimization of a loss functional. The loss expectation is defined as the risk associated to the stochastic scenario assumed. In this context, the final goal is that of minimizing the risk, looking for the socalled Bayesian solution (or decision) to the problem. Once a probabilistic scenario is assumed, the optimization task reduces to the minimization of a related loss expectation.

The difficulty with exploiting this framework is both theoretical and computational. The latter is due to the fact that the evaluation of the loss expectation involves the numerical integration of expensive simulation outputs, with respect to uncertain quantities. The former can also be easily understood: in a more standard MDO formulation (as well as in standard deterministic numerical optimization), all the relevant variables, parameters and functions are defined from a deterministic viewpoint and, apparently, the optimization process does not involve any kind of stochastic variation. The resulting optimal solutions are therefore likely specialized for the specific scenario assumed. Nevertheless, the performances of the final design may significantly drop in off-design conditions, when the deterministic assumptions used no longer hold. In this context, we look for a robust solution to the MDO problem, i.e., a solution able to perform well on average, in the whole probabilistic scenarios. The effects of properly considering the uncertainty, mainly consist in a loss in specialization and a gain in robustness. The MDO problem, re-formulated to take into account uncertainty, becomes a Multidisciplinary Robust Design Optimization (MRDO) problem. The aim of the present work is to analyze the combined effects of considering several disciplines under uncertainty in ship design problems, developing a MRDO procedure that utilizes efficient methods for uncertainty analysis and encompasses the features of the MDO framework. Theory and applications of MDO subject to uncertainty may be found in, e.g., Agarwal et al. (2004), Du and Chen (2000a, b, 2002), Giassi et al. (2004), Mavris et al. (1999), Smith and Mahadevan (2005), and Sues et al. (1995).

Here, the MRDO focuses on the stochastic variation of the operating conditions. The probability density function associated to the operating scenario is taken as a design requirement, and the expectation of the relevant merit factors is assessed during the optimization task. For solving the resulting minimization problem, a Particle Swarm Optimization (PSO) algorithm is used. The method, first introduced by Kennedy and Eberhart (1995) is here applied in the form proposed by Campana et al. (2009).

The application studied in this work consists in the optimization of a keel fin of a sailing yacht. The keel fin provides the side force able to contrast the wind, allowing the yacht to travel along directions not aligned with the wind itself. The keel sustains an heavy ballast bulb, and the 
bending moment arising in this configuration, together with the hydrodynamic loads, generate an elastic deformation, which cannot be ignored in the computation of the hydrodynamic performances. As a consequence, a fully coupled hydroelastic problem is considered. The solution of the deterministic problem has been illustrated in Campana et al. (2006). In this paper, a MRDO problem will be formulated and solved, considering a probabilistic sailing scenario, defined in terms of uncertain yaw angle.

The paper is organized as follows. The next section presents the general context of optimization problems affected by uncertainty. Then, in Section 3, Bayes principle is exploited to formulate the present problem for RDO. Section 4 presents a comparison between RDO and deterministic multi-point optimization. The general framework of MDO is presented in Section 5, whereas the "robust" extension of MDO to MRDO is given in Section 6. The numerical results are presented in Section 7 and the concluding remarks are given in Section 8.

\section{Design optimization subject to uncertainty}

In this Section, an overview of design optimization affected by uncertainty is presented. In this context, the designer concern is that of finding an optimal configuration able to keep a good performance under variation of some uncertain parameters. In order to achieve such an optimal solution, an optimality criterion, based on robustness of the final design, has to be defined. We remark that here, the term "robust" is always associated with the uncertainty of parameters. Therefore, attention to robustness always involves care to handle some kind of uncertainty. A number of authors in the literature give different meanings to robustness depending on the application, and different kind of uncertainties are addressed. The interested reader is referred to Beyer and Sendhoff (2007), Park et al. (2006) and Zang et al. (2005).

In order to define the context of the present work, the following standard-deterministic optimization problem is considered:

$$
\begin{array}{ccl}
\underset{\mathbf{x} \in X}{\operatorname{minimize}} & f(\mathbf{x}, \mathbf{y}), & \text { given } \mathbf{y}=\hat{\mathbf{y}} \in Y \\
\text { subject to } & g_{n}(\mathbf{x}, \hat{\mathbf{y}}) \leq 0, & n=1, \ldots, N \\
& h_{m}(\mathbf{x}, \hat{\mathbf{y}})=0, & m=1, \ldots, M
\end{array}
$$

where $\mathbf{x} \in \mathbf{X} \subseteq \mathbb{R}^{k}$ is the design variables vector (which represents the designer choice), $\hat{\mathbf{y}} \in Y \subseteq \mathbb{R}^{h}$ is the design parameters vector (which collects those parameters independent of the designer choice, e.g., environmental or operating conditions defining the scenario), and $f, g_{n}, h_{m}$ : $\mathbb{R}^{k+h} \rightarrow \mathbb{R}$, are respectively the optimization objective and the inequality and equality constraint functions. While handling the above problem, the following uncertainties may occur - the interested reader is also referred to Diez and Peri (2010a).

(a) Uncertain design variable vector When translating the designer choice into the "real-life" world, the design variables may be affected by uncertainties due to manufacturing tolerances or actuator precision. Assume a specific designer choice $\mathbf{x}^{*}$ and define as $\boldsymbol{u} \in U$ the error or tolerance related to this choice. ${ }^{1}$ We may assume $\boldsymbol{u}$ as a stochastic process with probability density function $p(\boldsymbol{u})$; by definition it is $\int_{U} p(\boldsymbol{u}) d \boldsymbol{u}=1$. The expected value of $\mathbf{x}^{*}$ is, therefore,

$\overline{\mathbf{x}^{*}}:=\mu\left(\mathbf{x}^{*}+\boldsymbol{u}\right)=\int_{U}\left(\mathbf{x}^{*}+\boldsymbol{u}\right) p(\boldsymbol{u}) d \boldsymbol{u}$.

Note that, in general, the probability density function $p(\boldsymbol{u})$ depends on the specific designer choice $\mathbf{x}^{*}$. It is clear that, if the stochastic process $\boldsymbol{u}$ has zero expectation, i.e.

$\overline{\boldsymbol{u}}:=\mu(\boldsymbol{u})=\int_{U} \boldsymbol{u} p(\boldsymbol{u}) d \boldsymbol{u}=0$

we obtain $\overline{\mathbf{x}^{*}}=\mathbf{x}^{*}$.

(b) Uncertain environmental and operating conditions In "real-life" applications, environmental and operational parameters may differ from the design conditions $\hat{\mathbf{y}}$, see Problem (1). The design parameters vector may be assumed as a stochastic process with probability density function $p(\mathbf{y})$ and expected value or mean

$\overline{\mathbf{y}}:=\mu(\mathbf{y})=\int_{Y} \mathbf{y} p(\mathbf{y}) d \mathbf{y}$.

Note that, in this formulation, the uncertainty on the operating conditions is not related to the definition of a specific design point. Environmental and operating conditions are treated as "intrinsic" stochastic processes in the whole domain of variation $Y$. For this reason, we do not define an error in the definition of the operating conditions, preferring the present approach which identifies the environmental and operational parameters in terms of their probabilistic distributions in the whole domain of variation.

(c) Uncertain evaluation of the functions of interest The evaluation of the functions of interest (objective and constraints) may by affected by uncertainty due to inaccuracy in modeling or computing. Collect objective and constraints in a vector $\mathbf{f}:=\left\{f, g_{1}, \ldots, g_{N}, h_{1}, \ldots, h_{M}\right\}^{T}$, and assume

\footnotetext{
${ }^{1}$ The symbol * is used in the present formulation to denote a specific
} designer choice. 
that the assessment of $\mathbf{f}$ for a specific "deterministic" design point, $\mathbf{f}^{*}:=\mathbf{f}\left(\mathbf{x}^{*}, \hat{\mathbf{y}}\right)$, is affected by a stochastic error $\boldsymbol{w} \in W$. Accordingly, the expected value of $\mathbf{f}^{*}$ is

$\overline{\mathbf{f}^{*}}:=\mu\left(\mathbf{f}^{*}+\boldsymbol{w}\right)=\int_{W}\left(\mathbf{f}^{*}+\boldsymbol{w}\right) p(\boldsymbol{w}) d \boldsymbol{w}$.

Note that, in general, the probability density function of $\boldsymbol{w}$, i.e. $p(\boldsymbol{w})$, depends on $\mathbf{f}^{*}$ and, therefore, on the design point $\left(\mathbf{x}^{*}, \hat{\mathbf{y}}\right)$.

Combining the above uncertainties, we may define the expected value of $\mathbf{f}$ as

$$
\begin{aligned}
\overline{\mathbf{f}}:=\mu(\mathbf{f})= & \iint_{U Y} \int_{W}\left[\mathbf{f}\left(\mathbf{x}^{*}+\boldsymbol{u}, \mathbf{y}\right)+\boldsymbol{w}\right] \\
& p(\boldsymbol{u}, \mathbf{y}, \boldsymbol{w}) d \boldsymbol{u} d \mathbf{y} d \boldsymbol{w}
\end{aligned}
$$

where $p(\boldsymbol{u}, \mathbf{y}, \boldsymbol{w})$ is the joint probability density function associated to $\boldsymbol{u}, \mathbf{y}, \boldsymbol{w}$. It is clear that $\overline{\mathbf{f}}=\overline{\mathbf{f}}\left(\mathbf{x}^{*}\right)$; in other words, the expectation of $\mathbf{f}$ is a function of the only designer choice. Moreover, the variance of $\mathbf{f}$ with respect to the variation of $\boldsymbol{u}, \mathbf{y}, \boldsymbol{w}$ is

$$
\begin{aligned}
V(\mathbf{f}):=\sigma^{2}(\mathbf{f})= & \iint_{U} \int_{W}\left\{\left[\mathbf{f}\left(\mathbf{x}^{*}+\boldsymbol{u}, \mathbf{y}\right)+\boldsymbol{w}\right]-\overline{\mathbf{f}}\left(\mathbf{x}^{*}\right)\right\}^{2} \\
& p(\boldsymbol{u}, \mathbf{y}, \boldsymbol{w}) d \boldsymbol{u} d \mathbf{y} d \boldsymbol{w}
\end{aligned}
$$

resulting, again, in a function of the designer choice variables. It may be noted that, here, $\{\cdot\}^{2}$ stands for the elementwise square, and $\mu(\mathbf{f})$ and $\sigma^{2}(\mathbf{f})$ are vectors of dimension $1+N+M$. The evaluation of the integrals in (6) and (7) is often referred as Uncertainty Quantification, UQ (Najm 2009; Iaccarino 2008; Mousaviraad et al. 2011).

With respect to the uncertainties outlined above, different approaches may be followed for the recasting of the optimization problem. Specifically, the optimization task may be defined in terms of:

- minimization of the variance, or of the standard deviation, $\sigma:=\sqrt{V}$, of $f$ : this leads to a robust design in a strict sense-e.g., Taguchi methods (Taguchi 1986);

- minimization of the expectation of $f$ : if $f$ represents a performance loss, then the expected value of $f$ can be seen as a risk-Bayesian approach as in statistical decision theory (De Groot 1970; Trosset et al. 2003; Kugele et al. 2008)—see next section;

- minimization of $f$ in the worst possible case; this is the most conservative approach- "minmax" approach (Trosset et al. 2003; Kugele et al. 2008);

- assessing probabilistic constraints in the minimization of the objective function (Tu et al. 1999; Sues et al.
2001; Du and Chen 2000b; Agarwal 2004; Agarwal and Renaud 2004).

With respect to the previous approaches, different definitions may be found in the literature - the interested reader is referred, again, to Beyer and Sendhoff (2007):

- Robust design (RD): process of defining the robust design in the strict sense (e.g., Taguchi methods). The attention in this case is mainly on variance or standard deviation.

- Robust optimization or Robust design optimization (RDO): optimization process considering uncertainties in the evaluation of the objective function; expected value, variance, worst case, etc. may be taken into account.

- Reliability-based design optimization (RBDO): the attention is focused on the statistical feasibility of the design (i.e., on the constraints). The constraints are treated as probabilistic inequalities and give a statistical feasible region.

While RD and RDO are mainly focused on expectation and variance of a cost function (Zang et al. 2005), the RBDO concentrates on handling the uncertainty of the constraints (Tu et al. 1999; Sues et al. 2001; Du and Chen 2000b; Agarwal 2004; Agarwal and Renaud 2004). The latter are treated as probabilistic inequalities (Nocedal and Wright 1999) and the $n$-th deterministic constraint of the type $g_{n}(\mathbf{x}, \mathbf{y}) \leq 0$ is treated using the general probabilistic statement

$P_{S}:=P\left[g_{n}(\mathbf{x}, \mathbf{y}) \leq 0\right], \quad P_{S} \geq P_{0}$,

where $P_{S}$ is the probability of success, $P[A]$ denotes the probability of the event $A$ and $P_{0}$ is a given target probability. Note that the probability of failure, $P_{F}$, equals $1-P_{S}$. In the following, the constraints of the optimization problem will be defined in the worst possible case, choosing a conservative approach $\left(P_{0}=1\right)$. The issues connected to the probabilistic handling of the constraints are beyond the scope of the present work, and will not be further addressed here.

\section{Decision making under uncertain operating conditions: robust design optimization through Bayes principle}

In this section, specific attention is paid to the uncertainty related to the environmental and operating conditions. In the context of naval applications, environmental and operating conditions may be considered as "intrinsic" stochastic 
functions, whose expected values and standard deviations can neither be influenced by the designer nor by the manufacturer. For this reason, assessing probabilistic operating conditions, may be interpreted as a relevant step towards a more comprehensive design optimization, bringing into focus "real-life" applications.

Assume that the optimization objective in Problem (1) is associated to a loss function (like, for instance, the performance loss with respect to a given target). Under the hypothesis of uncertain environmental and operating conditions, we may refer to $f(\mathbf{x}, \mathbf{y})$ as the loss associated to the designer choice $\mathbf{x}$, when the condition $\mathbf{y}$ occurs. Therefore, the expectation of the loss $f$, evaluated through the integral of (6) (limited to uncertain parameters $\mathbf{y}$, thus referring to uncertainty of type $b$ only), may be defined as the risk associated to the decision $\mathbf{x}$ under the distribution $p(\mathbf{y})$ (De Groot 1970). It follows that the designer should choose, if possible, a decision $\mathbf{x}$ which minimizes the risk (expected loss). Specifically, if we consider the Bayes risk, i.e. the lower bound of the expected loss for all the possible choices in $X$,

$\rho:=\inf _{\mathbf{x} \in X} \bar{f}=\inf _{\mathbf{x} \in X} \mu(f)$

we look for the Bayes decision of the problem, considering the distribution $p(\mathbf{y})$, i.e. the decision for which the risk equals the Bayes risk $\rho$. Therefore, the optimal designer choice is that which minimizes the expected loss of the system performances with respect to the stochastic variation of the environmental and operating conditions in $\mathbf{y}$ (Bayes principle; see, e.g. Trosset et al. 2003 and Kugele et al. 2008). It may be noted that in the present context, the design specifications are no longer given in terms of a single operating design point, but in terms of probability density function of the operating scenario (see also Diez et al. 2010).

It may be noted that the Bayesian approach to the decision problem may be enriched by considering, as a second objective function, the standard deviation of $f$. The latter extension possibly improves the robustness of the final design to the operating conditions variation.

The Bayesian approach to the designer decision problem (encompassing the standard deviation of $f$, if required) may be formulated as follows:

$$
\begin{aligned}
\underset{\mathbf{x} \in X}{\operatorname{minimize}} & f_{1}(\mathbf{x}):=\mu[f(\mathbf{x}, \mathbf{y})] \\
\text { and } & f_{2}(\mathbf{x}):=\sigma[f(\mathbf{x}, \mathbf{y})] \\
\text { subject to } & \sup _{\mathbf{y} \in Y}\left\{g_{n}(\mathbf{x}, \mathbf{y})\right\} \leq 0, \quad n=1, \ldots, N \\
& \mu\left[h_{m}(\mathbf{x}, \mathbf{y})\right]=0, \quad m=1, \ldots, M
\end{aligned}
$$

where, $\mu(f)$ and $\sigma(f)$ are given, by definition, through the UQ:

$\mu[f(\mathbf{x}, \mathbf{y})]:=\int_{Y} f(\mathbf{x}, \mathbf{y}) p(\mathbf{y}) d \mathbf{y}=\bar{f}(x)$

$\sigma^{2}[f(\mathbf{x}, \mathbf{y})]:=\int_{Y}[f(\mathbf{x}, \mathbf{y})-\bar{f}]^{2} p(\mathbf{y}) d \mathbf{y}=\sigma^{2}(x)$.

\section{Robust design optimization versus multi-point optimization}

The aim of this section is to provide the reader with a comparison between the RDO approach-handling probabilistic operating conditions - and the multi-point optimization approach.

A standard deterministic method to manage different operating conditions within an optimization problem is the use of an aggregate objective function (AOF) combining the same objective, evaluated for different operating pointsmulti-point optimization, e.g. Reuther et al. (1999a, b), Mirzaei et al. (2007) and Jameson et al. (2007). The latter may be expressed as

$f_{A O F}(\mathbf{x}):=\sum_{k=1}^{n} w_{k} f\left(\mathbf{x}, \mathbf{y}_{k}\right)$

where $w_{k} \in \mathbb{R}^{+}$. If, on one hand, this approach is able in principle to cope with different operating scenarios, on the other hand, the definition of the objective function in terms of $\mathbf{y}_{k}$ and $w_{k}$ depends on designer experience and sensitivity, resulting in an arbitrary choice. Moreover, no information about performance variation is available to the optimizer.

To compare the above approach to an RDO process managing uncertain operating conditions, advantage is taken of the numerical evaluation of the integral of (11). Specifically, using a Gaussian quadrature scheme for the integration of the objective $f$ over the operating conditions domain, the following approximation holds

$\mu(f):=\int_{Y} f(\mathbf{x}, \mathbf{y}) p(\mathbf{y}) d \mathbf{y} \approx \sum_{h=1}^{m} v_{h} f\left(\mathbf{x}, \mathbf{y}_{h}\right) p\left(\mathbf{y}_{h}\right)$

where the $v_{h}$ and the $\mathbf{y}_{h}$ represent respectively the Gaussian weights and abscissas, specifically assessed for the domain $Y$. It is apparent that the latter provides a particular multi-point AOF, with uniquely defined points and weights. Assuming $n=m$, comparing (13) and (14) gives

$w_{k}=v_{k} p\left(\mathbf{y}_{k}\right), \quad k=1, \ldots, n$ 
with $\mathbf{y}_{k}$ corresponding to the $k$-th Gaussian abscissa. It may be observed that the performance expectation $\mu(f)$, evaluated through quadrature, gives a uniquely defined AOF in the context of multi-point optimization.

\section{Multidisciplinary Design Optimization}

The basic elements of the MDO problem can be easily summarized. In particular, we report here a general overview which may help the reader to capture the main issues and difficulties related to MDO formulations of real problems.

Some specific aspects must be considered when attempting to identify reliable MDO formulations. Among them we can address the following ones:

- several disciplines are involved in order to provide the overall formulation;

- $\quad$ each discipline has to be considered essential for the overall formulation;

- the overall formulation can be hardly given as a nonlinear mathematical program on the whole;

- each discipline may be regarded as an independent problem with its own formulation. Theoretical results relative to each discipline (e.g. optimality conditions, sensitivity analysis, convergence analysis), solution techniques (e.g. solution methods, heuristics, etc.), and possibly codes, can be hardly worth also for the other disciplines;

- there is at least one iterative procedure such that

(a) at any step it computes the results provided by each discipline

(b) it yields at any step an intermediate result of the MDO formulation, where the independent disciplines are suitably gathered and coordinated.

Observe that the steps (a) and (b) are critical, and in general they do not suggest a unique nonlinear formulation for the overall MDO problem.

As usually assumed in multidisciplinary literature, we address as design unknowns some variables of the overall MDO formulation, included in the formulations of the disciplines, since they have a physical meaning. On the other hand, the formulation associated with each discipline can include also state unknowns (e.g. discipline auxiliary parameters, state unknowns of control systems, variables generated by the discretization of a PDE solver, etc.). Unlike design unknowns the latter variables are not given a specific physical meaning.

Now, let us consider the $p$ disciplines $D_{i}, i=1, \ldots, p$, with the vector $\left\{\mathbf{x}_{i}^{T}, \mathbf{s}_{i}^{T}\right\}^{T} \in \mathbb{R}^{n_{i}+m_{i}}$ associated with $D_{i}$. The vector $\mathbf{s}_{i} \in \mathbb{R}^{m_{i}}$ is the state of the $i$-th discipline $D_{i}$, while $\mathbf{x}_{i} \in \mathbb{R}^{n_{i}}$ is the design vector of $D_{i}$. Including also the sub-vector $\mathbf{x}_{0} \in \mathbb{R}^{n_{0}}$ of design unknowns, shared by the $p$ disciplines, we can provide a more formal definition of MDOformulations where: $\mathbf{x}=\left\{\mathbf{x}_{0}^{T} \mathbf{x}_{1}^{T} \cdots \mathbf{x}_{p}^{T}\right\}^{T} \in \mathbb{R}^{n}, n=$ $n_{0}+n_{1}+\cdots+n_{p}$ (design variables) and $\mathbf{s}=\left\{\mathbf{s}_{1}^{T} \cdots \mathbf{s}_{p}^{T}\right\}^{T} \in$ $\mathbb{R}^{m}, m=m_{1}+\cdots+m_{p}$ (state vector). In order to provide a general and formal definition for MDO formulations, we need to introduce the following assumption.

Assumption 1 Let us consider a real multidisciplinary problem, where the $p$ disciplines $D_{i}, i=1, \ldots, p$ are involved. Let $\mathbf{x}=\left\{\begin{array}{llll}\mathbf{x}_{0}^{T} & \mathbf{x}_{1}^{T} & \cdots & \mathbf{x}_{p}^{T}\end{array}\right\}^{T} \in \mathbb{R}^{n}, n=n_{0}+n_{1}+$ $\cdots+n_{p}$, and $\mathbf{s}=\left\{\mathbf{s}_{1}^{T} \cdots \mathbf{s}_{p}^{T}\right\}^{T} \in \mathbb{R}^{m}, m=m_{1}+\cdots+m_{p} ;$ suppose that the following hold:

1. for each discipline $D_{i}$ the set $B_{i} \subseteq \mathbb{R}^{n_{0} \times n_{i} \times m}$ (feasible set of $D_{i}$ ) exists such that

$$
\begin{aligned}
B_{i}= & \left\{\left(\mathbf{x}_{0}, \mathbf{x}_{i}, \mathbf{s}\right), \mathbf{x}_{0} \in \mathbb{R}^{n_{0}}, \mathbf{x}_{i} \in \mathbb{R}^{n_{i}}, s \in \mathbb{R}^{m}:\right. \\
& \left.g_{i}\left(\mathbf{x}_{0}, \mathbf{x}_{i}, \mathbf{s}\right) \geq 0, \quad A_{i}\left(\mathbf{x}_{0}, \mathbf{x}_{i}, \mathbf{s}\right)=0\right\} ;
\end{aligned}
$$

2. for each discipline $D_{i}$ the nonlinear map $f_{i}\left(x_{0}, x_{i}, s\right)$ exists, with $f_{i}: \mathbb{R}^{n_{0}} \times \mathbb{R}^{n_{i}} \times \mathbb{R}^{m} \rightarrow \mathbb{R}^{q_{i}}$, such that the formulation associated with the discipline $D_{i}$ is

$$
\min _{\left(\mathbf{x}_{0}, \mathbf{x}_{i}, s\right) \in B_{i}} f_{i}\left(\mathbf{x}_{0}, \mathbf{x}_{i}, \mathbf{s}_{i}\right), \quad i=1, \ldots, p
$$

( $f_{i}$ is constant in case $D_{i}$ represents a feasibility problem);

3. there exist the explicitly or implicitly defined maps $f(\mathbf{x}, \mathbf{s})=\varphi\left[f_{1}\left(\mathbf{x}_{0}, \mathbf{x}_{1}, \mathbf{s}\right), \ldots, f_{p}\left(\mathbf{x}_{0}, \mathbf{x}_{p}, \mathbf{s}\right)\right], g_{0}(\mathbf{x}, \mathbf{s})$, where $f: \mathbb{R}^{n} \times \mathbb{R}^{m} \rightarrow \mathbb{R}^{q}$ and $g_{0}: \mathbb{R}^{n} \times \mathbb{R}^{m} \rightarrow \mathbb{R}$, such that if $B=\left\{(\mathbf{x}, \mathbf{s}), \mathbf{x} \in \mathbb{R}^{n}, s \in \mathbb{R}^{m}: g_{0}(\mathbf{x}, \mathbf{s}) \geq\right.$ $\left.0,\left(\mathbf{x}_{0}, \mathbf{x}_{i}, \mathbf{s}\right) \in B_{i}, i=1, \ldots, p\right\}$, the overall real problem can be formulated as

$$
\min _{(\mathbf{x}, \mathbf{s}) \in B} f(\mathbf{x}, \mathbf{s}) \text {. }
$$

Definition 1 Suppose the Assumption 1 holds. Then, we say that Problem (16) is a Nonlinear MDO formulation for the MDO problem.

Though the Assumption 1 may apparently be considered an unnecessary theoretical overload, it provides a road map to distinguish between tractable MDO problems (i.e. for which a nonlinear MDO formulation exists), and hardly tractable MDO problems (i.e. for which a nonlinear MDO formulation is difficult to be identified).

Also in case the Assumption 1 holds (i.e. the problem in hand is a tractable MDO problem), then (16) often cannot be handled as a standard nonlinear program, on the overall. That is because the feasible set $B$ in (16) includes the so called MultiDisciplinary Analysis (MDA)

$M D A=\left\{\begin{array}{c}A_{1}\left(\mathbf{x}_{0}, \mathbf{x}_{1}, \mathbf{s}\right)=0 \\ \vdots \\ A_{p}\left(\mathbf{x}_{0}, \mathbf{x}_{p}, \mathbf{s}\right)=0 .\end{array}\right.$ 
MDA only implicitly describes a nonlinear system of equations, in as much as the $i$-th block of equalities $A_{i}\left(\mathbf{x}_{0}, \mathbf{x}_{i}, \mathbf{s}\right)=0$ may not correspond exactly to a set of nonlinear equations. $A_{i}\left(\mathbf{x}_{0}, \mathbf{x}_{i}, \mathbf{s}\right)=0$ may indeed be a black-box or it often corresponds to the discretization of PDE systems. This implies that the implicit function theorem cannot be exploited to explicitly compute the map $\mathbf{s}=\mathbf{s}(\mathbf{x})$ (and so reducing the number of unknowns).

In addition, the reader is warned that depending on the codes used to approach the solution of (16), some theoretical cares on handling the constraints must be paid. In particular, when Lagrangian or Penalty methods are adopted, specific assumptions on constraint qualification have to be satisfied, so that Lagrange multipliers exist and Karush-Kuhn-Tucker systems admit a solution (it is not difficult to find simple examples of MDO problems where the latter assumptions do not hold at all). Hence, this proves the intrinsic difficulty of providing a suitable formulation, say Problem (16), such that efficient algorithms can be adopted and possibly a complete convergence analysis holds. A more complete characterization of different reformulations for an MDO problem is given in Campana et al. (2007a). The latter reference also includes a taxonomy on MDO problems for ship design, based on both theoretical and algorithmic perspectives, in order to address an MDO formulation.

In this paper, in order to simplify the notation and to emphasize our context of ship design optimization subject to uncertain operating conditions, we assume that each discipline is based on a specific disciplinary analysis (from simple algebraic formulas to complex PDEs), that may be schematically depicted as an input-output relation in Fig. 1.

Moreover, in our context, the input of each discipline reduces to a set of deterministic design variables, $\mathbf{x}:=$ $\left\{\mathbf{x}_{i}^{T}, \mathbf{x}_{S}^{T}\right\}^{T}$, uncertain operating parameters, $\mathbf{y}:=\left\{\mathbf{y}_{i}^{T}, \mathbf{y}_{S}^{T}\right\}^{T}$, and a set of parameters supplied by other disciplines, $\left\{\mathbf{a}_{j}\right\}_{j \neq i}:=\left\{\mathbf{a}_{1}^{T}, \ldots, \mathbf{a}_{i-1}^{T}, \mathbf{a}_{i+1}^{T}, \ldots, \mathbf{a}_{n}^{T}\right\} ;$ the analysis produces a set of outputs, $\mathbf{a}_{i}$. The system-level design variables, $\mathbf{x}_{S}$ and the system level operating parameters $\mathbf{y}_{S}$, are those shared by all the disciplines. The disciplinary design variables, $\mathbf{x}_{i}$ are assumed local to $\Delta_{i}$ as well as the disciplinary operating parameters $\mathbf{y}_{i}$.

The disciplinary analysis has often the functional form $\mathbf{a}_{i}=A_{i}\left(\mathbf{x}_{i}, \mathbf{y}_{i}, \mathbf{x}_{S}, \mathbf{y}_{S}, \mathbf{a}_{1}, \ldots, \mathbf{a}_{i-1}, \mathbf{a}_{i+1}, \ldots, \mathbf{a}_{n}\right)$, where $A_{i}$ is assumed to be independent of $A_{j}, \forall j \neq i$. In the context of the MDO problem, the coupled Multidisciplinary Analysis (MDA) system reflects the physical requirement that a

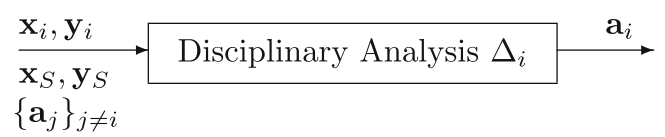

Fig. 1 Discipline analysis $\Delta_{i}$ of the $i$-th discipline

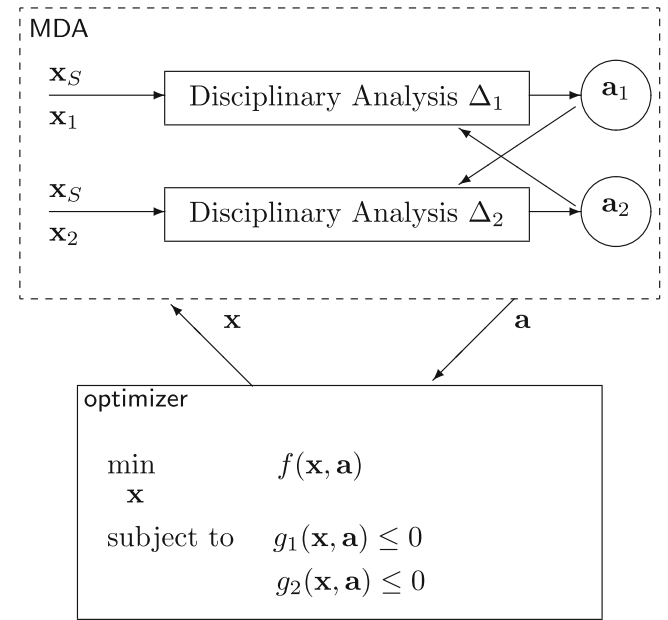

Fig. 2 MDO procedure

solution simultaneously satisfies all the disciplinary analyses. The multidisciplinary analysis system, in the explicit form, is therefore given by the simultaneous system of equations:

$\left\{\begin{array}{l}\mathbf{a}_{1}=A_{1}\left(\mathbf{x}_{S}, \mathbf{y}_{S}, \mathbf{x}_{1}, \mathbf{y}_{1}, \mathbf{a}_{2}, \ldots, \mathbf{a}_{n}\right) \\ \mathbf{a}_{2}=A_{2}\left(\mathbf{x}_{S}, \mathbf{y}_{S}, \mathbf{x}_{2}, \mathbf{y}_{2}, \mathbf{a}_{1}, \mathbf{a}_{3}, \ldots, \mathbf{a}_{n}\right) \\ \vdots \\ \mathbf{a}_{n}=A_{n}\left(\mathbf{x}_{S}, \mathbf{y}_{S}, \mathbf{x}_{n}, \mathbf{y}_{n}, \mathbf{a}_{1}, \ldots, \mathbf{a}_{n-1}\right)\end{array}\right.$

Solving the fully coupled system of (17) leads to a full multidisciplinary analysis. The solution is in this case a consistent solution that satisfies all the disciplines.

Up to now we have just looked for a multidisciplinary equilibrium among the disciplines. The most natural MDO problem formulation is to impose an optimizer over the MDA (17) and find the optimal solution with respect to the deterministic designer choice $\mathbf{x}$. Figure 2 presents, at a glance, a deterministic two-disciplines MDO procedure, where no uncertainties are considered during the optimization.

\section{Multidisciplinary Robust Design Optimization}

The extension of the above procedure, to take into account the application of Bayes principle to an uncertain operating scenario, involves the integration of the objective $f$ over the uncertain parameters domain. It may be noted that the uncertainties propagate in the MDA framework. With respect to each discipline involved, the uncertainty related to the definition of the input variables and parameters may be referred as external, whereas the source of uncertainty related to the analysis tool itself (e.g., inaccuracy in computing) is addressed as internal (Du and Chen 2002). As a 


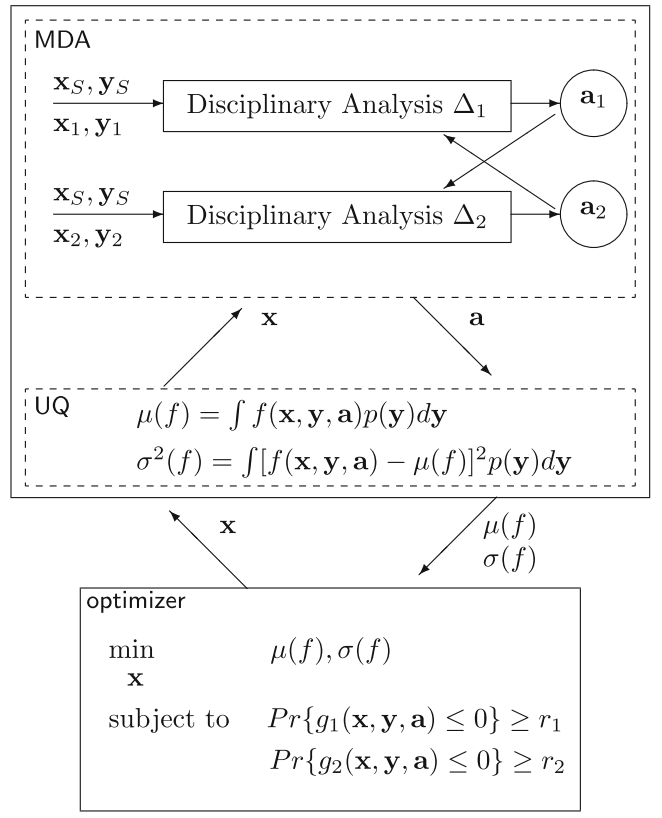

Fig. 3 MRDO procedure

result, the final multidisciplinary equilibrium is affected by uncertainty.

As clearly appears, the solution of the Multidisciplinary Robust Design Optimization problem represents an expensive task, due to the fact that the integrals of (11) and (12) apply to a function supplied by the multidisciplinary equilibrium of (17), for every value of the uncertain parameters $\mathbf{y}$. It is worth noting that the standard deterministic MDO scheme involves coupling an optimization algorithm (optimizer, see Fig. 2) with the MDA framework. Taking into account the uncertainty in that context (thus formulating the MRDO problem) requires the insertion between the optimization algorithm and the MDA, of an UQ scheme (as summarized in Fig. 3), resulting in a more expensive computational problem.

Of course the computation of $\mu(f)$ and $\sigma^{2}(f)$ in (11) and (12) depends on the MDO final formulation adopted, as suggested by relation (16). On this guideline, Alexandrov and Lewis (2002) and Campana et al. (2007a) clearly indicate that Assumption 1 might be often satisfied using a wide variety of reformulations, each providing a different balance between a theoretical perspective and a computational perspective. In other terms, a specific MDO formulation might be preferable when theoretical issues like constraint qualification conditions at solution are a keypoint. ${ }^{2}$ On the other hand, the flip-side is that the same formulation may be not suitable due to its computational burden. The latter

\footnotetext{
${ }^{2}$ The reader is warned that for instance, in many real-life problems, the Mangasarian-Fromowitz constraint qualification condition (MFCQ, e.g., Nocedal and Wright 1999) does not hold at the solution points, the latter being a critical issue for the algorithm adopted.
}

complexity for the choice of the MDO formulation turns to hold similarly also for MRDO, e.g., multilevel formulations might partially simplify the computation, but they suffer for poor theoretical properties when non-convex functions are involved in the formulation.

In addition, once a formulation for MDO is chosen, the MRDO may be solved by means of surrogate models and approximations. These may be used as functions of the design variables and/or uncertain parameters. Although surrogate models for design optimization and UQ are often very fruitful and dramatically abate the computational effort, their use is beyond the scope of the present work and, therefore, no further addressed. The interested reader is addressed to, e.g., Alexandrov and Lewis (2001), Peri and Campana (2005), Allaire and Willcox (2010) and Mousaviraad et al. (2011).

\section{Numerical results}

In this section, we present numerical results obtained through the formulation for MRDO. The application consists in the optimization of the performance of a keel fin of a sailing yacht. The keel fin provides the hydro-dynamic side force, balancing the wind-force and, thus, allowing the yacht to travel along directions not aligned with the wind itself. The keel sustains an heavy ballast bulb, allowing for the equilibrium of the roll moment. The bending moment along the fin, originated by concentrated and hydrodynamic loads, yields an elastic deformation, which cannot be ignored in the computation of the hydrodynamic performances. As a consequence, a fully coupled hydroelastic problem is considered. The multidisciplinary analysis system consists in solving together the two disciplines, hydrodynamic $\left(\Delta_{1}\right)$ and structural analysis $\left(\Delta_{2}\right)$. Figures 4 and 5 show at a glance the hydrodynamics and structural solutions, respectively. The models used and the algorithms

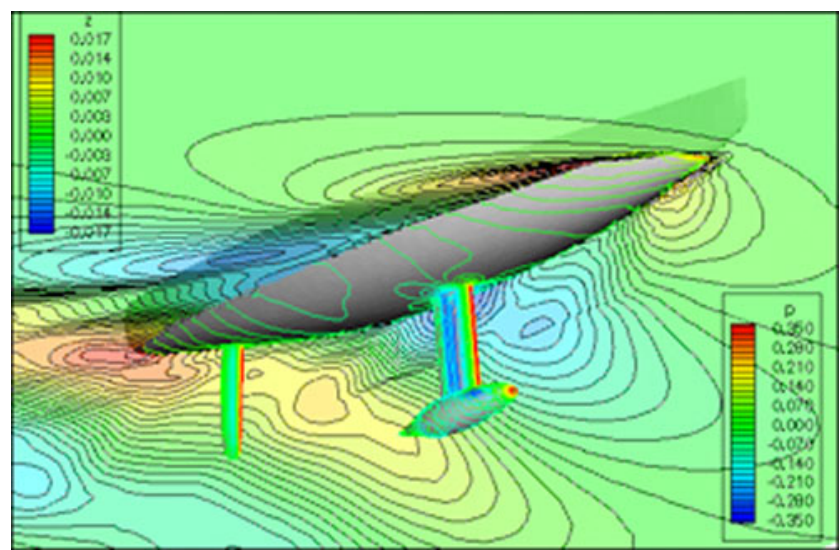

Fig. 4 Hydrodynamics $\left(\Delta_{1}\right)-C F D$ solution for the pressure field 
Fig. 5 Structural analysis $\left(\Delta_{2}\right)$-FEM solution for the elastic displacements of the fin

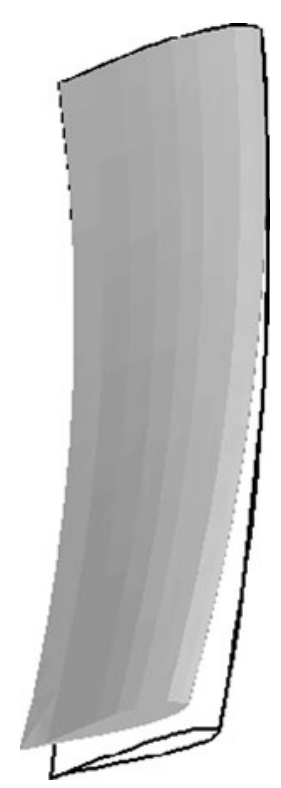

adopted to solve the multidisciplinary equilibrium for the deterministic case has been illustrated in Campana et al. (2006) and, therefore, not repeated here. The sailing operating conditions adopted here are the following. The cruise speed equals 10 knots, the heel angle is fixed at 30 degrees. The stochastic operating scenario is taken into account in terms of the probability density function (PDF) of the yaw angle, which is used in this numerical test as the only uncertain parameter (see Table 1). Problem (10) is taken considering purely the Bayes principle as defined in Trosset et al. (2003) and Kugele et al. (2008), and illustrated by Trosset et al. (2003) in the optimization of an airfoil shape for minimum drag, varying the Mach number. This approach is acceptable when (as in the present case) the original objective represents a performance measure not related to safety. Accordingly, the expectancy measures of robustness (Beyer and Sendhoff 2007), or Bayes risk (De Groot 1970), is assumed as the only optimization goal, therefore looking for the Bayesian decision (De Groot 1970). Extensions of the present approach to robust multi-objective optimization aimed at minimum average performance and performance variation may be found in, e.g., Beyer and Sendhoff (2007) and Diez and Peri (2010a). Here, the optimization objective is defined as the expectation of the fin efficiency (lift over drug, $C_{L} / C_{D}$ ) over the probabilistic operating scenario.

The design variables used pertain to the fin geometry (see Fig. 6). Specifically, seven design variables are used to

Table 1 Probabilistic operating scenario

\begin{tabular}{llll}
\hline $\begin{array}{l}\text { Uncertain } \\
\text { parameter }\end{array}$ & $\begin{array}{l}\text { Lower } \\
\text { bound }\end{array}$ & $\begin{array}{l}\text { Upper } \\
\text { bound }\end{array}$ & $\begin{array}{l}\text { Distribution } \\
\text { type }\end{array}$ \\
\hline Yaw angle & $1^{\circ}$ & $9^{\circ}$ & Uniform \\
\hline
\end{tabular}

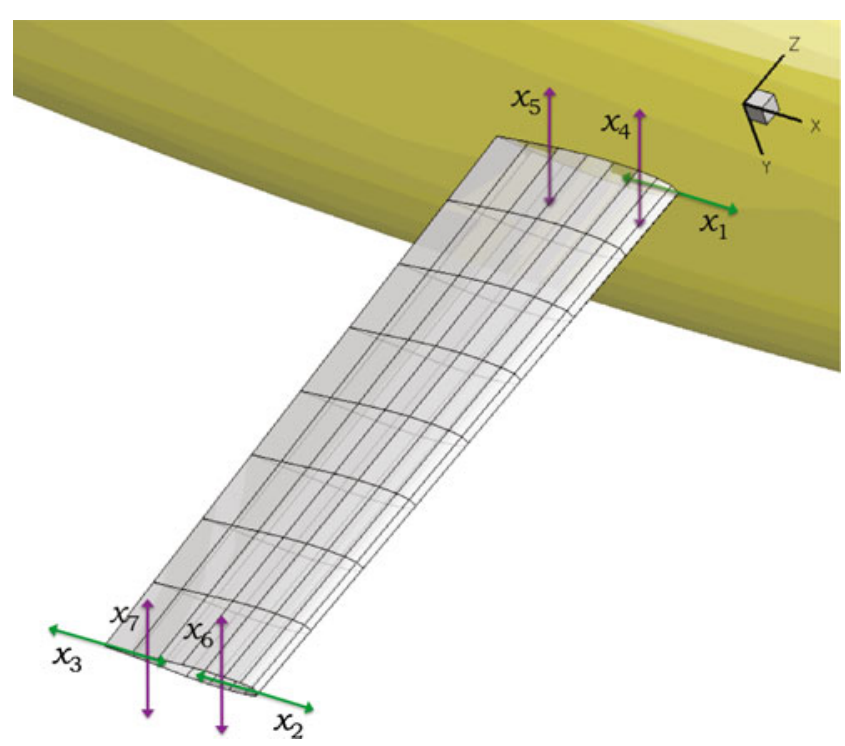

Fig. 6 Schematic representation of the seven design variables used for the fin deformation (planform and hydrofoil)

change the overall shape of the fin. The original unmodified fin is defined as a full body. Three design variables are used to modify the fin planform. Specifically, $x_{1}, x_{2}, x_{3}$, control the leading edge at the root, at the tip and the trailing edge at the fin tip, respectively. Only longitudinal deformations are allowed. The fin section is shaped using two hydrofoils, one at the fin root and the second at the fin tip. The section varies accordingly and linearly along the span. The modification of the sections (root and tip) is provided by four variables that move two control points for each section $\left(x_{4}, x_{5}, x_{6}, x_{7}\right.$, respectively). The sections are forced to be symmetric and only transversal deformations are allowed.

The results are organized as follows. First, a parametric analysis of the fin efficiency, varying the yaw angle, is

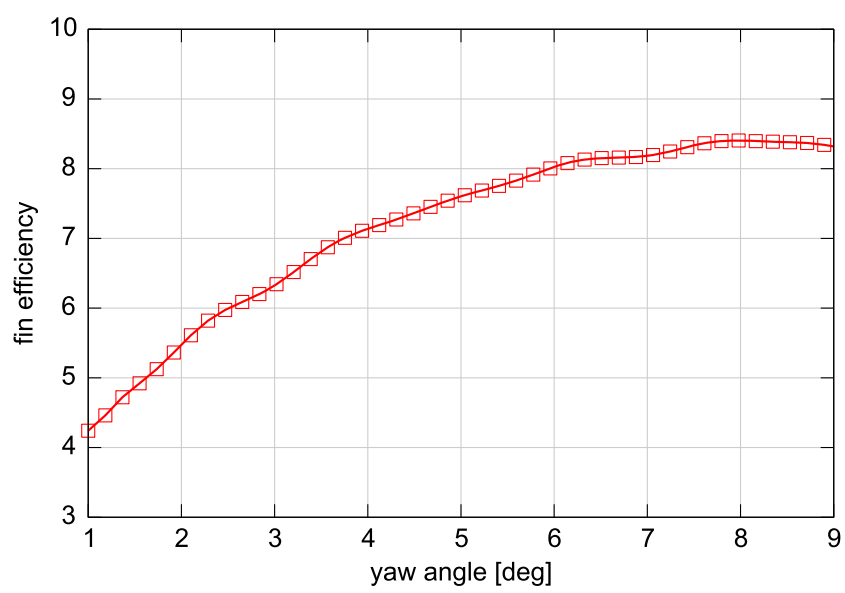

Fig. 7 Parametric analysis-fin efficiency vs. yaw angle for the original configuration 


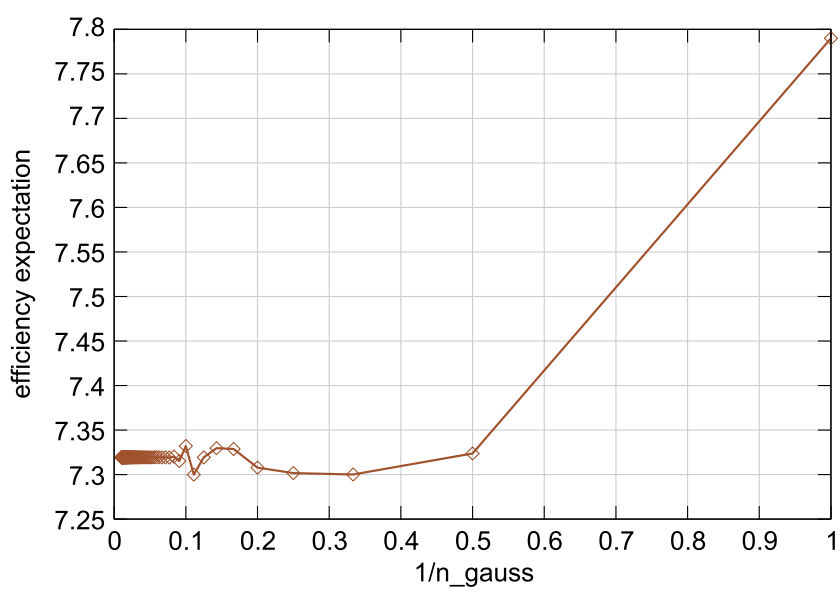

Fig. 8 Uncertainty quantification-convergence of the GaussLegendre integration for the expectation of the fin efficiency

conducted through a fully-coupled multidisciplinary analysis. Then, a convergence analysis for the Uncertainty Quantification (UQ) is given and commented. Finally, the optimization procedure is presented for both deterministic and stochastic approach.

\subsection{Uncertainty Quantification (UQ)}

In order to assess the objective function(s) for robust design, the evaluation of the integrals in (11) and (12) (Uncertainty Quantification, UQ) has to be performed. As a result, we get the expectation (and, if required, the standard deviation) of the performance measure adopted. Having in mind that during a complete optimization procedure, the evaluation of the objective function may be required thousands of times, and that the assessment of the objective function involves the integration over the uncertain-parameter-domain of the

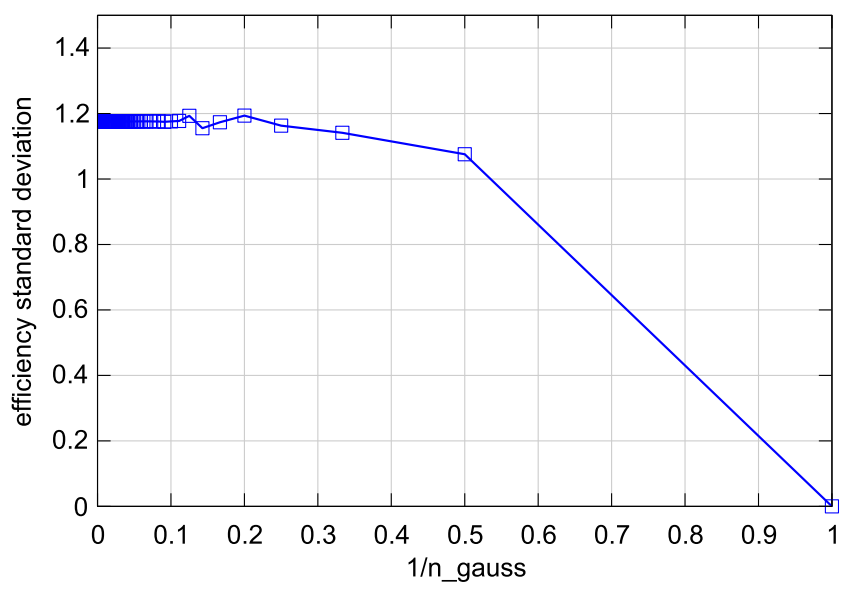

Fig. 9 Uncertainty quantification-convergence of the GaussLegendre integration for the standard deviation of the fin efficiency

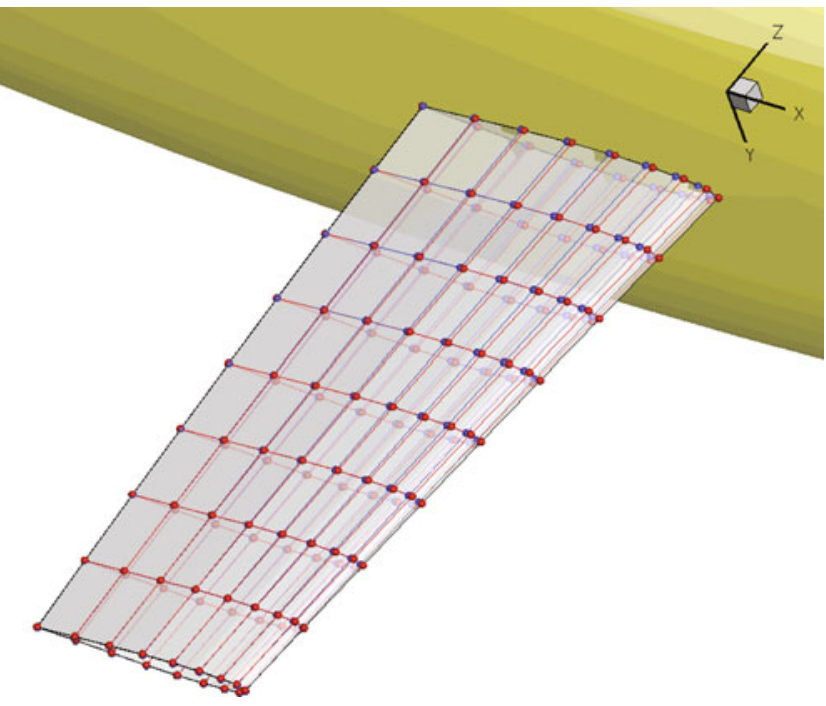

Fig. 10 A comparison between MDO (red) and MRDO (blue) solutions

performance measure, it is easy to understand that a compromise between accuracy and computational efficiency represents a critical issue.

For the original fin configuration, a parametric study for the efficiency, obtained by varying the yaw angle, is performed through MDA and presented in Fig. 7. In order to compute the integral of (11) and, if required, (12), a GaussLegendre quadrature is used and a convergence analysis is shown in Figs. 8 and 9. Specifically, the evaluation of expectation and standard deviation of the fin efficiency is shown, varying the number of Gaussian points.

Focusing on the evaluation of the fin efficiency expectation, Figs. 8 and 9 reveal that a number of 11 or more Gaussian abscissas would be necessary to reach the convergence in the evaluation of the integrals of (11) and (12).

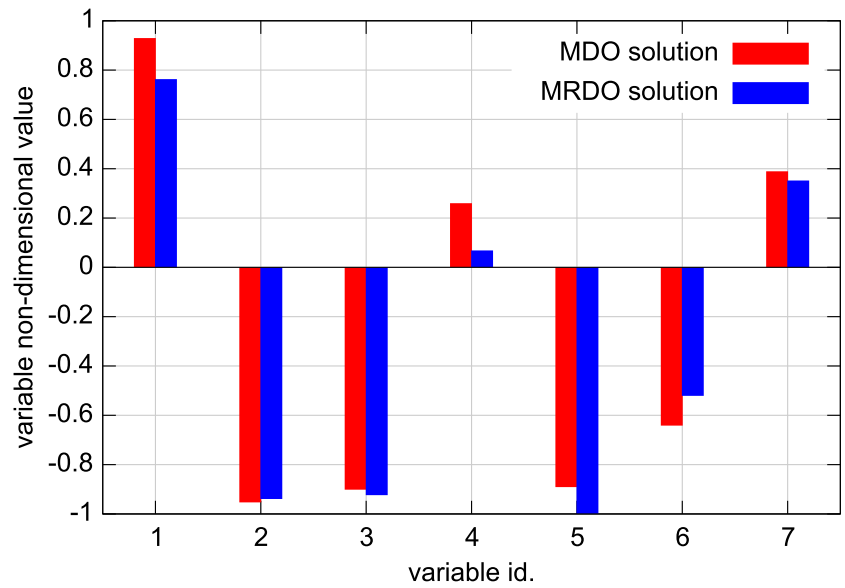

Fig. 11 A comparison between MDO- and MRDO-optimal solutions: variables non-dimensional value $(-1$ indicates the lower bound, whereas +1 indicates the upper bound) 
Table 2 Optimal design variables

\begin{tabular}{lrr}
\hline $\begin{array}{l}\text { Design } \\
\text { variable id. }\end{array}$ & $\begin{array}{l}\text { Deterministic } \\
\text { MDO }\end{array}$ & \multicolumn{1}{l}{$\begin{array}{l}\text { Stochastic } \\
\text { MRDO }\end{array}$} \\
\hline 1 & 0.9298 & 0.7632 \\
2 & -0.9531 & -0.9394 \\
3 & -0.9015 & -0.9237 \\
4 & 0.2602 & 0.0686 \\
5 & -0.8912 & -0.9994 \\
6 & -0.6417 & -0.5211 \\
7 & 0.3895 & 0.3521 \\
\hline
\end{tabular}

Accordingly, a number of 11 Gaussian points are used for the following numerical results.

It may be noted that, here, the uncertainty is on one of the input of the analysis models. This allow for a non-intrusive approach to the UQ. In other words, the analysis codes may be seen as a black box, without the need of modifying their source codes; the UQ is done by embedding the deterministic analysis tool in an integration scheme. Issues related to deterministic and stochastic convergence and validation of non-intrusive UQ methods are addressed in Mousaviraad et al. (2011).

\subsection{Multidisciplinary Robust Design Optimization (MRDO)}

In this subsection, the results of the stochastic MRDO procedure (aimed at fin efficiency expectation) are presented and compared to those obtained through a deterministic MDO process. The latter is defined by considering the uncertain parameter (yaw angle) equal to its expectation (i.e. $\left.5^{\circ}\right)$. The probabilistic operating scenario is that shown in Table 1.

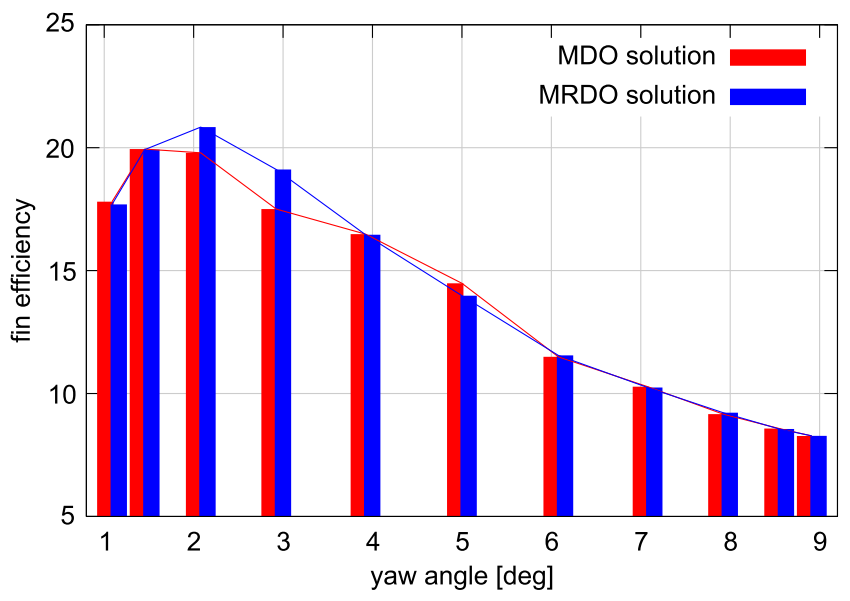

Fig. 12 A comparison between MDO- and MRDO-optimal solutions performance: fin efficiency vs. yaw angle
Fig. 13 Pressure field for the MDO solution

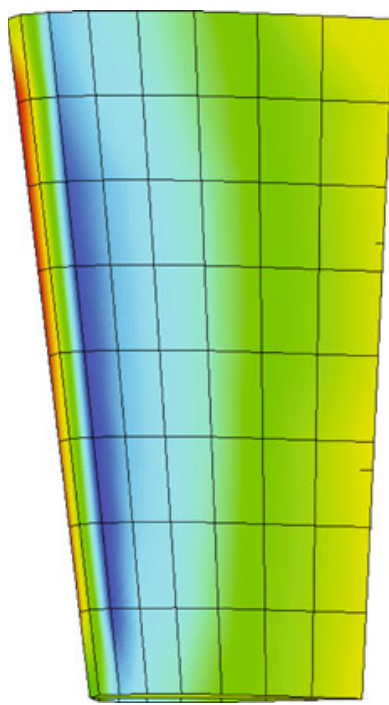

For MRDO, a particle swarm optimization (PSO) algorithm (Campana et al. 2009) is imposed over the UQ, whereas for deterministic MDO, the same algorithm is imposed over the MDA. For both approaches, a maximum number of 3,000 objective function evaluations is assumed. The results are summarized in Fig. 10 and 11 and Table 2. It may be noted how the optimal solutions for MDO and MRDO fall in two different points of the design space.

Moreover, Fig. 12 present a parametric analysis for the fin efficiency, varying the yaw angle (evaluated at the Gaussian points). It may be noted how the deterministic solution (MDO) has a greater value for the efficiency when the yaw angle equals its expectation (specialized solution), whereas the stochastic solution (MRDO) presents the best overall behavior (robust solution). Although the differences are not large, the last result is worthy of attention since

Fig. 14 Pressure field for the MRDO solution

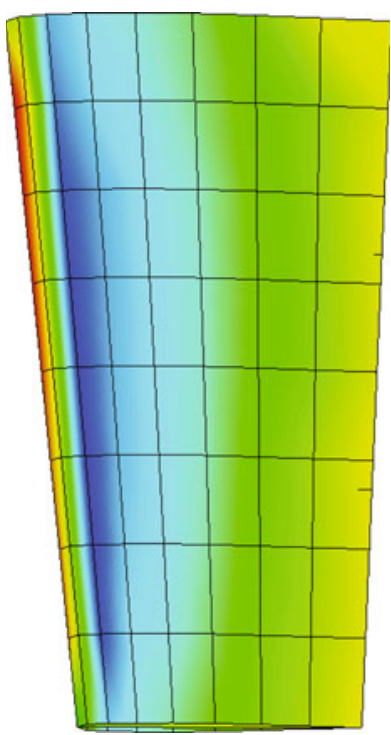


Fig. 15 Pressure field for the original configuration

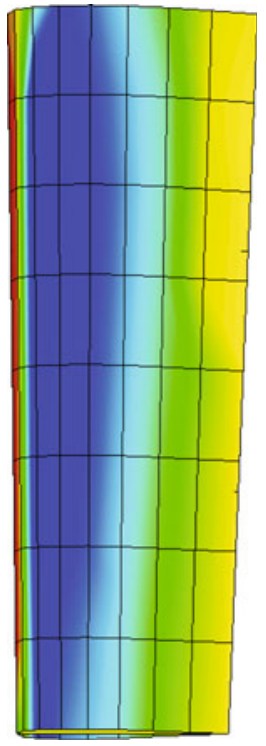

demonstrates the consistency of the formulation and the numerical implementation.

Finally, Figs. 13 and 14 present the CFD solution for the pressure field for MDO- and MRDO-optimal solutions respectively. The pressure field for the original configuration is presented in Fig. 15. In the present analysis, the bulb is considered as a concentrated mass, and thus not explicitly appears in the CFD solution. Even though the differences are small, some considerations can be made. The MRDO-optimal solution has a greater bending stiffness, due to larger thickness (greater absolute values for $\left.x_{4}, x_{6}, x_{7}\right)$ and slightly larger chords $\left(x_{1}\right)$. This results in a lower bending and a greater efficiency at low yaw angles $\left(2^{\circ}, 3^{\circ}\right)$.

\section{Concluding remarks}

A formulation for Multidisciplinary Robust Design Optimization (MRDO) has been presented and applied to the optimization of a keel fin of a sailing yacht, subject to uncertain operating conditions. The present formulation couples the multidisciplinary analysis (MDA) with the uncertainty quantification (UQ) over the stochastic parameters domain. The Bayesian solution to the design problem is pursued by imposing an optimizer over the UQ.

In the present context, the design specifications are no longer given in terms of a single operating design point, but in terms of probability density function of the operating scenario.

The numerical results presented here reveal that the formulation for stochastic MRDO drives the optimizer towards a solution different to the deterministic MDO result. Moreover, the MRDO optimal solution is characterized by a better overall performance in the stochastic operating scenario. Despite of the fact that in this work only seven design variables are used, with a limited number of function evaluations, the MDO- and MRDO-optimal solutions obtained are different and the performances are consistent with the formulation. The authors believe that using a greater number of design variables (thus widening the research space) with a greater number of function evaluations, allows for a more conspicuous dissimilarity between deterministic- and stochastic-optimal solutions. Moreover, the stochastic operating scenario should include additional parameters such as heel and pitch angles and speed, so as to handle a more realistic case.

\section{References}

Allaire D, Willcox K (2010) Surrogate modeling for uncertainty assessment with application to aviation environmental system models. AIAA J 48:1791-1803

Alexandrov NM, Lewis RM (2001) An overview of first-order model management for engineering optimization. Optim Eng 2:413-430

Alexandrov NM, Lewis RM (2002) Analytical and computational aspects of collaborative optimization for multidisciplinary design. AIAA J 40:301-309

Agarwal H (2004) Reliability based design optimization: formulations and methodologies. PhD dissertation, Univ of Notre Dame, South Bend, IN, USA

Agarwal H, Renaud J (2004) Reliability based design optimization using response surfaces in application to multidisciplinary systems. Eng Optim 36:291-311

Agarwal H, Renaud J, Preston EL, Padmanabhan D (2004) Uncertainty quantification using evidence theory in multidisciplinary design optimization. Reliab Eng Syst Saf 85:281-294

Baumal AE, McPhee JJ, Calamai PH (1998) Application of genetic algorithms to the design optimization of an active vehicle suspension system. Comput Methods Appl Math 163:87-94

Beyer HG, Sendhoff B (2007) Robust optimization-a comprehensive survey. Comput Methods Appl Math 196:3190-3218

Campana EF, Fasano G, Peri D (2006) Issues on nonlinear programming for Multidisciplinary Design Optimization (MDO), in ship design frameworks. In: Mota Soares CA et al (eds) III European conference on computational mechanics solids, structures and coupled problems in engineering. Lisbon, Portugal

Campana EF, Fasano G, Peri D (2007a) Nonlinear programming approaches in the Multidisciplinary Design Optimization (MDO) of a sailing yacht keel Fin. In: 9th intl conf on numerical ship hydrodynamics, Ann Arbor, MI, USA

Campana EF, Peri D, Pinto A (2007b) Multiobjective optimization of a containership using deterministic particle swarm optimization. J Ship Res 51:217-228

Campana EF, Liuzzi G, Lucidi S, Peri D, Piccialli V, Pinto A (2009) New global optimization methods for ship design problems. Optim Eng 10:533-555

De Groot MH (1970) Optimal statistical decisions. McGraw Hill, New York

Diez M, Peri D (2009) Global optimization algorithms for robust optimization in naval design. In: 8 th intl conf on computer applications and information technology in the maritime industries, COMPIT 2009. Budapest, Hungary 
Diez M, Peri D (2010a) Robust optimization for ship conceptual design. Ocean Eng 37:966-977

Diez, M, Peri D (2010b) Two-stage stochastic programming formulation for ship design optimization under uncertainty. Ship Technol Res 57:172-181

Diez M, Peri D, Campana EF, Iemma U (2010) Robust decision making in aerial and marine vehicles optimization: a designer's viewpoint. Ent Risk Mgmt 2:68-86

Du X, Chen W (2000a) Methodology for managing the effect of uncertainty in simulation-based design. AIAA J 38:1471-1478

Du X, Chen W (2000b) Towards a better understanding of modeling feasibility robustness in engineering design. J Mech Des 122:385394

Du X, Chen W (2002) Efficient uncertainty analysis methods for multidisciplinary robust design. AIAA J 40:545-552

Giassi A, Bennis F, Maisonneuve, J-J (2004) Multidisciplinary design optimisation and robust design approaches applied to concurrent design. Struct Multidisc Optim 28:356-371

Hicks RM, Henne PA (1978) Wing design by numerical optimization. J Aircr 15:407-412

Iaccarino G (2008) Quantification of uncertainty in flow simulations using probabilistic methods. Non-equilibrium gas dynamics: from physical models to supersonic flights. In: VKI lecture series

Iemma U, Diez M (2006) Optimal conceptual design of aircraft including community noise prediction. In: Collection of technical papers-12th AIAA/CEAS aeroacoustics conference, vol 5, pp 2855-2864

Jameson A, Leoviriyakit K, Shankaran S (2007) Multi-point aerostructural optimization of wings including planform variations. In: 45th aerospace sciences meeting and exhibit, Reno, NV, USA

Kennedy J, Eberhart R (1995) Particle swarm optimization. In: IEEE intl conf on neural networks. Perth, Australia

Kodiyalam S, Sobieszczanski-Sobieski J (2001) Multidisciplinary design optimization - some formal methods, framework requirements, and application to vehicle design. Int $\mathrm{J}$ Veh Des 25: $3-22$

Kugele SC, Trosset MW, Watson LT (2008) Numerical integration is statistical decision-theoretic methods for robust design optimization. Struct Multidisc Optim 36:457-475

Marczyk J (2000) Stochastic multidisciplinary improvement: beyond optimization. In: 8th AIAA/USAF/NASA/ISSMO symposium on multidisciplinary analysis and optimization. Long Beach, CA, USA

Mavris DN, Bandte O, Delaurentis DA (1999) Robust design simulation: a probabilistic approach to multidisciplinary design. J Aircr 36:298-307

Mirzaei M, Hosseini SN, Roshanian J (2007) Single and multi-point optimization of an airfoil using gradient method. Aircr Eng Aerosp Tec 70:611-620

Mohammadi B, Pironneau O (2001) Applied shape optimization for fluids. Clarendon, Oxford

Morino L, Bernardini G, Mastroddi F (2006) Multi-disciplinary optimization for the conceptual design of innovative aircraft configurations. Comput Model Eng Sci 13:1-18

Mousaviraad MS, He W, Diez M, Stern F (2011) Framework for convergence and validation of stochastic UQ and relation- ship to deterministic V\&V. Int J Uncertainty Quantification. doi:10.1615/Int.J.UncertaintyQuantification.2012003594

Najm HN (2009) Uncertainty quantification and polynomial chaos techniques in computational fluid dynamics. Annu Rev Fluid Mech 41:35-52

Nocedal J, Wright W (1999) Numerical optimization. Springer, New York

Padula SL, Gumbert CR, Li W (2006) Aerospace applications of optimization under uncertainty. Optim Eng 7:317-328

Papanikolaou A (2010) Holistic ship design optimization. Comput Aided Des 42:1028-1044

Park GJ, Lee TH, Wang, KH (2006) Robust design: an overview. AIAA J 44:181-191

Parsons MG, Scott RL (2004) Formulation of multicriterion design optimization problems for solution with scalar numerical optimization methods. J Ship Res 48:61-76

Peri D, Campana EF (2003) Multidisciplinary design optimization of a naval surface combatant. J Ship Res 47:1-12

Peri D, Campana EF (2005) High-fidelity models in global optimization. Lect Notes Comput Sci 3478:112-126

Pinto A, Peri D, Campana EF (2004) Global optimization algorithms in naval hydrodynamics. Ship Technol Res 51:123-133

Ray T, Gokarn RP, Sha OP (1995) A global optimization model for ship design. Comput Ind 26:175-192

Reuther JJ, Jameson A, Alonso JJ, Rimlinger MJ, Saunders D (1999a) Constrained multipoint aerodynamic shape optimization using an adjoint formulation and parallel computers, part 1. J Aircr 36:5160

Reuther JJ, Jameson A, Alonso JJ, Rimlinger MJ, Saunders D (1999b) Constrained multipoint aerodynamic shape optimization using an adjoint formulation and parallel computers, part 2. J Aircr 36:6174

Smith N, Mahadevan S (2005) Integrating system-level and component-level designs under uncertainty. J Spacecr Rockets 42:752-760

Sobieszczanski-Sobieski J, Haftka RT (1997) Multidisciplinary aerospace design optimization: survey of recent developments. Struct Optim 14:1-23

Sues RH, Oakley DR, Rhodes GS (1995) Multidisciplinary stochastic optimization. Proc Eng Mech 2:934-937

Sues RH, Cesare MA, Pageau S, Wu JY-T (2001) Reliability-based optimization considering manufacturing and operational uncertainties. J Aerosp Eng 14:166-174

Taguchi G (1986) Quality engineering through design optimization. Krauss International Publications, White Plains

Trosset MW, Alexandrov NM., Watson, LT (2003) New methods for robust design using computer simulations. In: Proceedings of the section on physical and engineering sciences, American Statistical Association

Tu J, Choi KK, Park YH (1999) A new study on reliability-based design optimization. J Mech Des 121:557-564

Willcox K, Wakayama S (2003) Simultaneous optimization of a multiple-aircraft family. J Aircr 40:616-622

Zang C, Friswell MI, Mottershead JE (2005) A review of robust optimal design and its application in dynamics. Comput Struct $83: 315-326$ 